\title{
A Comprehensive Analysis for Extracting Single Diode PV Model Parameters by Hybrid GA-PSO Algorithm
}

\author{
C.Saravanan \\ Sr.Assistant Professor, \\ Department of Electrical \& Electronics Engg., \\ Tagore Engineering College,
}

\begin{abstract}
This paper proposes an improved model approach to single diode PV model by Hybrid Genetic Algorithm Particle Swarm Optimization (Hybrid GA-PSO) technique. The main objective is to extract accurate parameters of PV model for the simulator developers which could help them to develop an enhanced PV model in Matlab/Simulink environment. Specifications provided by the manufacturer's data sheet of PV module have been used for the computation of model parameters. Hybrid approach of GA and PSO is purposely chosen to utilize their effectiveness in these kinds of applications. This method is used to optimize the parameters Ipv, a, Rse and Rsh with their best optimal values. For the confirmation of accuracy and computational time of the proposed method, poly-crystalline PV module (KL070) has been selected and the best optimal value of each parameter has been obtained using Hybrid GA-PSO. Finally, the extracted values have been used in the single diode PV model and tested for various irradiations and temperatures. The proposed methodology results in marked improvement and can be a tool for simulator developers who require absolute and accurate model.
\end{abstract}

Keywords: Hybrid GA-PSO, Photo voltaic, poly crystalline, MATLAB.

\section{INTRODUCTION}

Renewable energy sources particularly photovoltaic are proven to be both clean and economical due to the new advanced technology and efficient cells. Solar energy is obviously environmentally advantageous relative to any other energy source and hence it is becoming popular. This increasing demand urges the simulator developers to model PV cells, so that it cloud help the Design Engineers to simulate and verify their solar based power systems. Modeling of PV cell involves identification of equivalent circuit parameters. Based on the type of PV model circuit, the circuit parameters may vary. To extract the exact parameters of the PV model there are various methods and like analytical method, optimization method, and iterative method. Among these methods, optimization methods are very effective to extract the parameters of PV model. Nowa-days researchers use single diode PV model for simulation purposes. Genetic Algorithms (GA) and Particle Swarm Optimization (PSO) are both population based algorithms that have proven to be successful in solving a variety of complex problems.

However, both methods have strengths and weaknesses. To extract parameters of single diode model GA is performed [1-3]. Two diode models are performed to extract parameter by Newton Raphson Iteration method [4].

\author{
M.A.Panneerselvam,Ph.D \\ Professor, \\ Department of Electrical \& Electronics Engg.,
}

MATLAB/SIMULINK is used for modeling Photo Voltaic $(\mathrm{PV})$ as performed in [5-11]. Characteristics evaluation and parameter extracted by experimental analysis has been done for solar array model [12]. Both Genetic Algorithm and Particle swarm optimization is used to extract parameter in single diode PV model [13]. Based on the survey, still researchers are doing in the field of hybridization with existing algorithms for the extraction of PV model parameters. So far, the proposed Hybrid GA-PSO has not been presented by any author before. However, based on accuracy, consistence and computation time the above approaches lack and fail to propose an accurate single diode PV model. This paper presents a novel hybrid GAPSO algorithm, combining the strengths of GA with those of PSO. This paper is organized as follows; Section 2 briefly describes the mathematical analysis of single diode model for PV simulation. Comprehensive analysis of the PV module using its current-voltage and power-voltage characteristics is presented in section 3. Detailed Procedure of Hybrid GA-PSO is presented with a flowchart in section 4. Section 5 presents the simulation results of the application of Hybrid GA-PSO. Finally comparison with standard GA is tabulated in section 5.2.

\section{SINGLE DIODE PV MODEL}

The single diode model that becomes the basis of evaluation is depicted in figure 1 . The output current of the model is well known and can be described as:

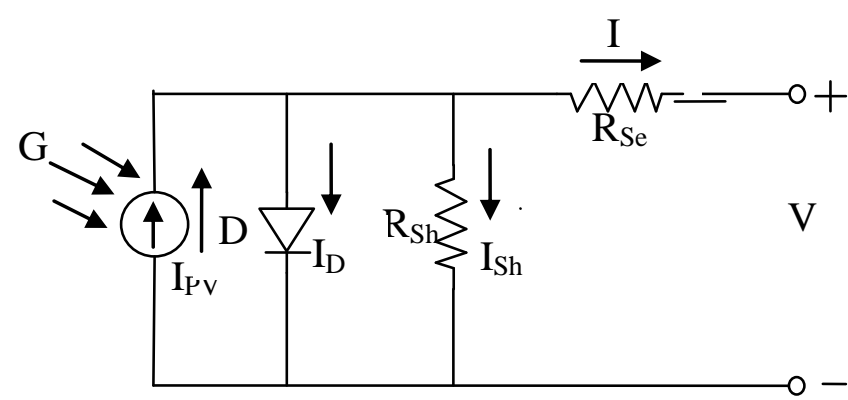

Fig.1 Equivalent circuit for single diode PV model

$I=I_{p v}-I_{o}\left[\exp \left(\frac{q\left(V+I R_{S e}\right)}{K T a}\right)-1\right]-\left[\frac{V+I R_{S e}}{R_{S h}}\right]$

where,

$\mathrm{I}_{\mathrm{pv}}=$ Current generated by the incident of light

$\mathrm{I}_{0}=$ Reverse saturation current of diode

$\mathrm{V}_{\mathrm{T}}=$ Thermal voltage $=N s K T / q$

$\mathrm{N}_{\mathrm{s}}=$ Number of cells connected in series

$\mathrm{q}=$ Electron charge $\left(1.60217646 \times 10^{-19} \mathrm{C}\right)$

$\mathrm{k}=$ Boltzmann constant $\left(1.3806503 \times 10^{-23} \mathrm{~J} / \mathrm{K}\right)$ 
$\mathrm{T}=$ temperature of the $\mathrm{p}-\mathrm{n}$ junction in Kelvin $a=$ diode ideality factor

Accurate PV model of this single diode type can be achieved only if the circuit parameters namely, photocurrent $I_{p v}$, diode ideality factor $a$, series resistance $R_{s e}$ and shunt resistance $R_{s h}$ are correctly chosen. Note that equation (1) is a transcendental equation which cannot be solved by direct analysis.

\section{COMPREHENSIVE ANALYSIS OF SINGLE DIODE PV MODEL}

In this section, methodology of single diode PV model and Hybrid GA-PSO objective function are described as follows;

\subsection{Methodology}

The single diode PV model parameters have been obtained as follows. Given a set of I-V data of a particular cell, the curve fitting process using GA and Hybrid GA-PSO is applied to update the values of model parameters in equation (1). To apply the optimization method, the equation (1) has to be re-written as:

(2)

$$
\begin{array}{r}
X(V, I, \phi)=I_{p v}-I_{o}\left(\exp ^{\frac{V+I_{s e}}{a+V_{T}}-}\right)- \\
\frac{V+I_{s e}}{R_{\text {sh }}}-I
\end{array}
$$

Where $\Phi=\left[I_{P V}, a, R_{s e}, R_{s h}\right]$ are the parameters set to be extracted. At the end of iteration, the values in $\Phi$ have been updated and then checked whether it has converged to the correct value. This procedure continues up to the intended maximum number of iterations.

\subsection{Fitness function for the extraction of PV model parameters}

To objectively evaluate the performance of this method, an objective function $Y$ is introduced. In this work, the objective function can be given by:

$Y=\sqrt{\sum_{m=1}^{n} X\left(V_{m}, I_{m}, \emptyset\right)^{2}}$

(3)

where $I_{m}$ and $V_{m}$ are the data pair of $\mathrm{I}-\mathrm{V}$ characteristic curve, respectively; hence, the extraction procedure is based on the minimization of $\mathrm{Y}$ in conjunction to $\Phi$. A smaller value of $\mathrm{Y}$ implies the least deviation between I and $\mathrm{V}$ data and this is computed by Hybrid GA-PSO algorithm. Ideally, a zero value of $\mathrm{Y}$ is desired.

\section{HYBRID GA-PSO}

Although GA has been successfully applied to various problems, using GA for large-scale optimization could be very expensive due to its requirement of a large number of function evaluations for convergence. This would result in a prohibitive cost for computation of function evaluations even with the best computational facilities available today. Considering the efficiency of the PSO and the compensatory property of GA and PSO, combining the searching abilities of both methods in one algorithm seems to be a logical approach. In this paper, the hybrid of GA and
PSO named Hybrid GA-PSO is presented. This algorithm consists of four major operators: enhancement, selection, crossover, and mutation. The flowchart of the Hybrid GAPSO is shown in figure 2 .

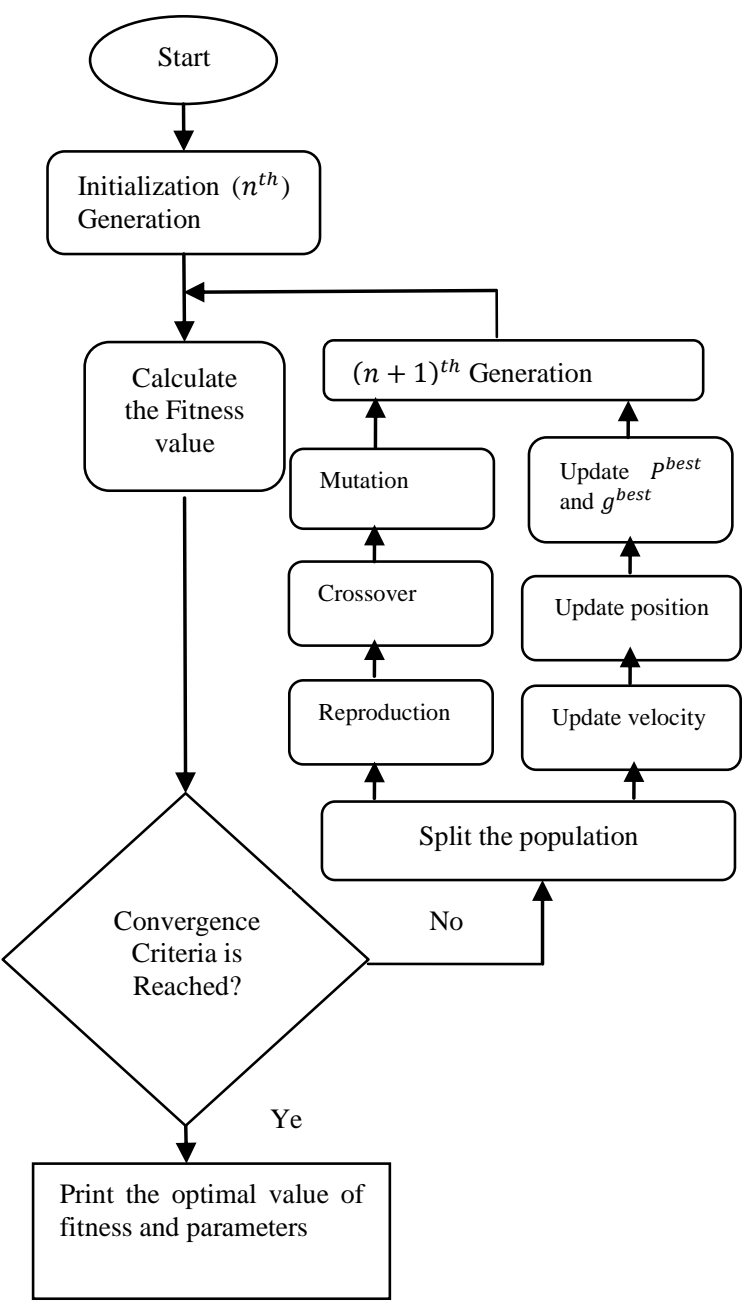

Fig. 2. Flow chart for Hybrid GA-PSO Algorithm

\section{SUMMARY AND CONCLUDING REMARKS}

In this paper, the simulation of PV model parameters using the proposed Hybrid GA-PSO method is done using MATLAB R2010b with Intel Core i3 CPU @ $2.53 \mathrm{GHz}$ processor, 3 GB RAM under windows 7 environment. The KL070 PV module is used for simulation study. The Parameters settings in Hybrid GA-PSO method are as follows; For GA: Generation-100, Population size-100, Selection-Roulette and Crossover- Arithmetic. For PSO: Cognito constant (C1)-0.5, Socio Constant (C2)-1.5 and Inertia constant $(\mathrm{w})-0.03$. The results obtained using the proposed Hybrid GA-PSO method is compared with GA algorithm in a judicial way.

An optimization search algorithm for improving the accuracy of the extracted values of PV model parameters using Hybrid GA-PSO algorithm has been implemented successfully. The results show that the use of hybrid genetic algorithm and particle swarm optimization for solar cell parameter extraction significantly decreases the error in the 
extracted values and hence improves the accuracy of the determined parameters.

Based on the proposed Hybrid GA-PSO approach presented in section 4, the convergence of the optimization is first investigated. The results generated from the proposed method are then validated with the manufacturer experimental data.

\subsection{Hybrid GA-PSO Convergence}

The KL070 PV module is used to study the Hybrid GAPSO convergence whose specifications are given in TABLE 1 . The result is obtained by executing the proposed method at the particular temperature for 25 times. It's quite clear that Hybrid GA-PSO performs the best in optimizing the objective function, for it is more possible to search the optima than other algorithms. Compared with GA, Hybrid GA-PSO could find better objective function value, which shows the effectiveness of chaotic local search. According to the result shown in TABLE 2, Hybrid GA-PSO tends to find the global optimum faster than other algorithms and hence has a higher convergence rate.

Table 1. Specification of PV model given by manufacturer

\begin{tabular}{|l|c|}
\hline \multicolumn{1}{|c|}{ Parameters } & Polycrystalline KL070 \\
\hline Pmax & $70 \mathrm{Wp}$ \\
Voc & $21.5 \mathrm{~V}$ \\
Vmpp & $17.1 \mathrm{~V}$ \\
Isc & $4.59 \mathrm{~A}$ \\
Impp & $4.10 \mathrm{~A}$ \\
Ns & 36 \\
Kv(-..36\%/k) & -0.0774 \\
Ki $(.06 \% / k)$ & $2.754 \mathrm{e}-3$ \\
\hline
\end{tabular}

Table 2. Fitness values for GA and Hybrid GA-PSO Algorithm

\subsection{Validating the Model}

To validate the model, the proposed Hybrid GA-PSO method is used to identify $I_{p v}, a, R_{s e}$ and $R_{s h}$ for KL070 PV module. The extracted parameters obtained using the method proposed here for the above module is given in TABLE 3. In the same table, the extracted parameters by GA method is also presented for comparison. Satisfactory agreement is obtained for most of the extracted parameters.

Table 3. Comparison of optimized values using GA and Hybrid GA-PSO

\begin{tabular}{|c|c|c|}
\hline Algorithm & GA & Hybrid GA-PSO \\
\hline Parameters & KL070 & KL070 \\
\hline$I_{p v}$ & 4.6 & 4.6 \\
\hline $\mathrm{R}_{\mathrm{se}}$ & .10 & .28 \\
\hline $\mathrm{R}_{\mathrm{sh}}$ & 108.62 & 97.88 \\
\hline $\mathrm{a}$ & 1.2429 & 1.2921 \\
\hline Computation time & 123.2 & 81.5 \\
\hline
\end{tabular}

To illustrate and verify the nonlinear $\mathrm{P}-\mathrm{V}$ and $\mathrm{I}-\mathrm{V}$ output characteristics of PV module, the PV model is simulated for different solar insolation and temperature conditions. Figures 3 and 4 shows the simulated P-V and I-V curves for different solar insolation conditions respectively. Similarly, figures 5 and 6 the same curves for different temperature conditions respectively.

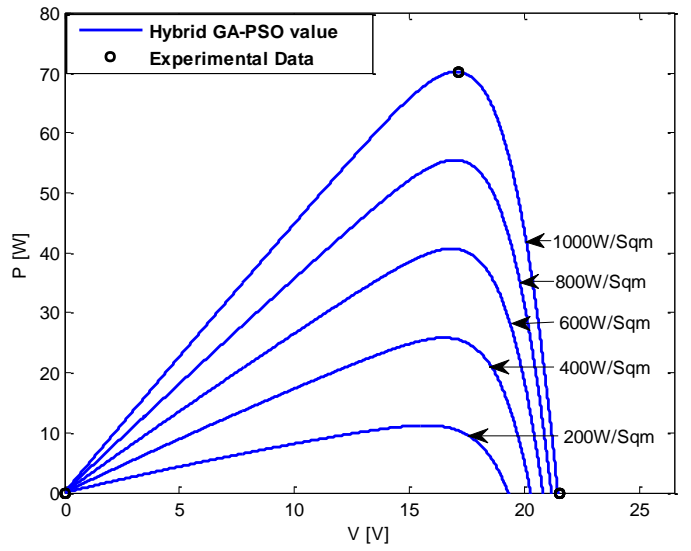

Fig 3: P-V Curve for different insolation

\begin{tabular}{|l|c|c|}
\hline \multicolumn{1}{|c|}{ Algorithm } & GA & Hybrid GA-PSO \\
\hline Fitness Value & KL070 & KL070 \\
\hline Best & $1.049 \times 10^{-6}$ & $2.42 \times 10^{-8}$ \\
Average & $8.649 \times 10^{-4}$ & $1.17 \times 10^{-4}$ \\
Worst & $5.42 \times 10^{-5}$ & $2.97 \times 10^{-5}$ \\
\hline
\end{tabular}

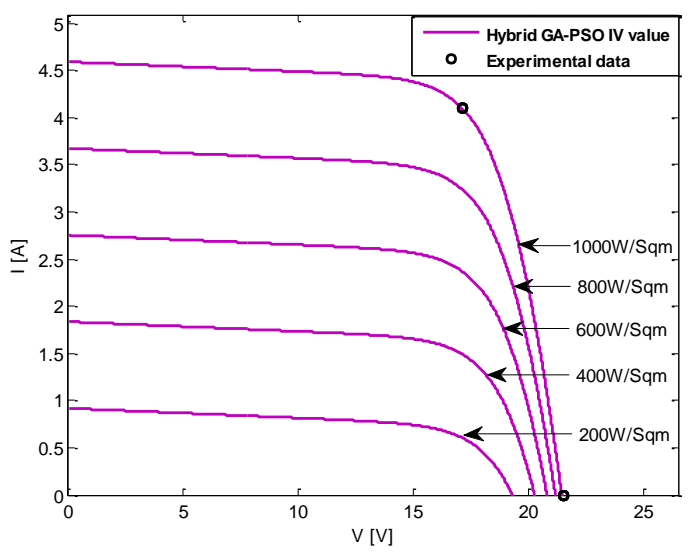

Fig 4: I-V Curve for different insolation 


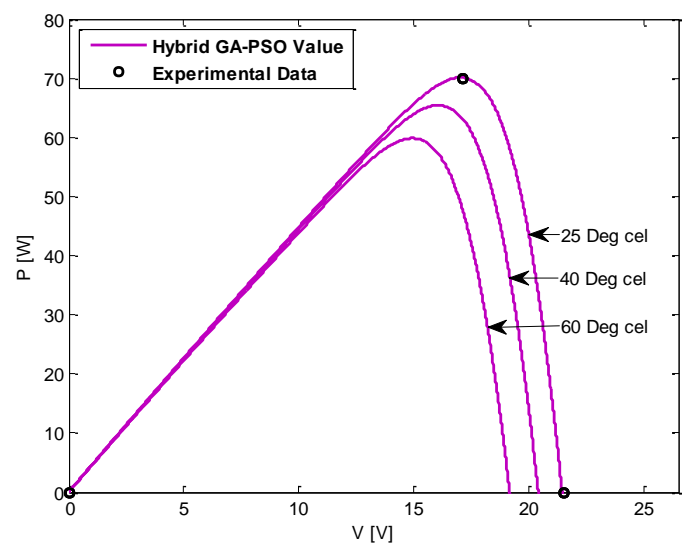

Fig 5: P-V Curve for different temperature

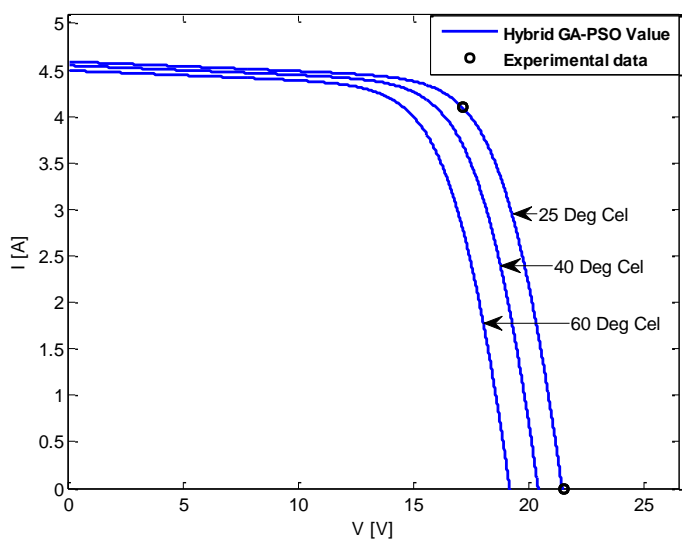

Fig 6: I-V Curve for different temperature

\section{CONCLUSION}

This paper proposed a powerful method of extracting solar cell parameters which directly affects the conversion efficiency, the power conversion and characteristics curve of the solar cell. Number of parameters to be extracted is limited to four parameters $I_{p v}, a, R_{s e}$, and $R_{s h}$. Hybrid GAPSO method has been successfully applied to the module KL070 under different temperatures and solar insolations. The results obtained are in good agreement with those published previously. This method is very simple to use. It allows real time characterization of different types of solar cells and modules under different environmental conditions. The entire procedure is very simple, practical and inexpensive.

\section{ACKNOWLEDGMENTS}

The author would like to acknowledge the help received from M. K. D. Ulaganathan and K.Karthikeyan.

\section{REFERENCES}

[1] Eftichios Koutroulis a,*, Dionissia Kolokotsa b ,AntonisPotirakis a , Kostas Kalaitzakis "Methodology for optimal sizing of stand-alone photovoltaic/windgenerator systems using genetic algorithms" a Elsevier Solar Energy 80 (2006) 1072-1088.

[2] Tina, G.M. Viale A. Doria n.6, Catania, 95125, Italy Richardson, Z.J.; Mahdi, A.J. "Thermal parameter identification of photovoltaic module using genetic algorithm", Renewable Power Generation
(RPG 2011), IET Conference Sept. 2011 University of Catania, Page(s): 1 - 6

[3] J K Maherchandani, Chitranjan Agarwal, Mukesh Sahi "Estimation of Solar Cell Model Parameter by Hybrid Genetic Algorithm Using MATLAB" International Journal of Advanced Research in Computer Engineering \& Technology (IJARCET)Volume 1, Issue 6, August 2012 ISSN: 2278 - 1323

[4] Kashif Ishaque, Zainal Salam, and Hamed Taheri "Accurate MATLAB Simulink PV System Simulator Based on a Two-Diode Model JPE 11-2-9 Journal of Power Electronics, Vol. 11, No. 2, March 2011

[5] Tarak Salmi*, Mounir Bouzguenda**, Adel Gastli**, Ahmed Masmoudi" "MATLAB/Simulink Based Modelling of Solar Photovoltaic Cell", INTERNATIONAL JOURNAL of RENEWABLE ENERGY RESEARCH Tarak Salmi et al., Vol.2, No.2, 2012

[6] Jing_Jun_Soon Sch. of Electr. \& Electron. Eng., Nanyang Technol. Univ., Singapore, Singapore KaySoon low "Photovoltaic Model Identification Using Particle Swarm Optimization with Inverse Barrier Constraint", Power_Electronics, IEEE Transactions on Date of Publication: Sept. 2012 Volume: 27, Issue:9, Page(s): 3975-3983

[7] Huan-Liang Tsai, Ci-Siang $\mathrm{Tu}$, and Yi-Jie $\mathrm{Su}$ Member, "Development of Generalized Photovoltaic Model Using MATLAB/SIMULINK". IAENG Proceedings of the World Congress on Engineering and Computer Science 2008 WCECS 2008, October 22 - 24, 2008, San Francisco, USA.

[8] Krisztina Leban, Ewen Ritchie Aalborg University, Pontoppidanstræde, Aalborg, "Selecting the Accurate Solar Panel Simulation Model", NORPIE/2008, Nordic Workshop on Power and Industrial Electronics, June 911,2008

[9] Sonal Panwara, Dr. R.P. Sainib "Development and Simulation of Solar Photovoltaic model using Matlab/simulink and its parameter extraction", International Conference on Computing and Control Engineering (ICCCE 2012), 12 \& 13 April, 2012.

[10] Horia Andrei, Costin Cepisca, Sorin Dan Grigorescu Traian Ivanovici1, Paul Andrei, "Modelling Of The Pv Panels Circuit Parameters Using The 4-Terminals Equations And Brune's Conditions", Scientific Bulletin Of The Electrical Engineering Faculty - Year 10 No. 1 (12).

[11] J. Surya Kumari* and Ch. Sai Babu** "Mathematical Modeling and Simulation of Photovoltaic Cell using Matlab-Simulink Environment", International Journal of Electrical and Computer Engineering (IJECE) Vol. 2, No. 1, February 2012, pp. 26-34 ISSN: 2088-8708

[12] Bidyadhar Subudhi* and Raseswari Pradhan** "Characteristics Evaluation and Parameter Extraction of a Solar Array based on Experimental Analysis", IEEE PEDS 2011, Singapore, 5 - 8 December 2011.

[13] Jing Jun Soon, Kay-Soon Low "Optimizing Photovoltaic Model Parameters for Simulation", 9781-4673-0158-9/12/2012 IEEE. 